\title{
SINTOMATOLOGÍA ASOCIADA A TRASTORNOS DE SALUD MENTAL EN TRABAJADORES SANITARIOS EN PARAGUAY: EFECTO COVID-19d
}

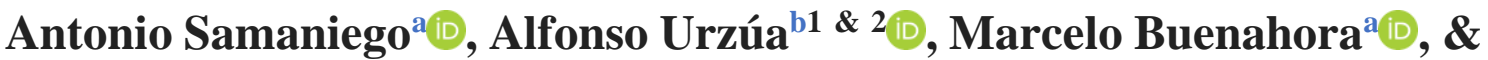 \\ Pablo Vera-Villarroel ${ }^{\mathrm{C}} \mathbb{}(\mathrm{A}$.
}

Universidad Nacional de Asunción, Asunción, Paraguay. ${ }^{\text {a }}$

Universidad Católica del Norte, Antofagasta, Chile. ${ }^{\mathrm{b}}$

Centro Análysis $I+D$, Santiago, Chile. ${ }^{\mathrm{c}}$

\begin{abstract}
RESUMEN
La enfermedad por COVID-19 no solo ha provocado un colapso del sistema sanitario y la muerte de miles de personas, sino que está repercutiendo en la salud de las personas que están brindando atención a los infectados. En este marco, el objetivo de esta investigación fue determinar prevalencias de depresión, ansiedad, insomnio, distrés y fatiga por compasión, así como factores relacionados a la presencia de sintomatología asociada a estos trastornos en profesionales de los equipos de salud. Para esto, se encuestó en formato online a 126 profesionales sanitarios del Paraguay, de los cuales el $83 \%$ son mujeres, con una edad promedio en la muestra total de 32 años. Se aplicaron los cuestionarios $P H Q-9$ para depresión, GAD7 para ansiedad, ISI-7 para insomnio, IES-R para distrés y el módulo de fatiga por compasión del ProQOL - CSF-vIV. Se encuentra que los porcentajes de participantes que reportaron síntomas clasificables como moderados y severos fueron de un 32.2\% para depresión, $41.3 \%$ en ansiedad, $27.8 \%$ en insomnio, 38.9\% en distrés y un $64.3 \%$ en fatiga por compasión. Adicionalmente las mujeres y los de menor edad presentaron un riesgo mayor de presentar síntomas. Los datos aportan evidencia a la necesidad de establecer estrategias de prevención o intervención para abordar los problemas de salud mental en los profesionales de la salud.
\end{abstract}

\section{Palabras Claves}

COVID-19; salud mental; trabajadores de la salud; ansiedad; depresión; distrés; insomnio

\begin{abstract}
COVID-19 disease has not only led to a collapse of the health system and the death of thousands of people but is also having an impact on the health of those who are providing care for those infected. In this context, the aim of this research was to determine prevalences of depression, anxiety, insomnia, distress and compassionate fatigue, as well as factors related to the presence of symptoms associated with these disorders in healthcare workers. For this, 126 health professionals from Paraguay were surveyed online, of which $83 \%$ were women, with an average age in the total sample of 32 years. The questionnaires PHQ-9 for depression, GAD-7 for anxiety, ISI-7 for insomnia, IES-R for distress and the module of compassion fatigue of ProQOL - CSF-vIV were applied. It was found that the percentages of participants who reported symptoms classified as moderate and severe were $32.2 \%$ for depression, $41.3 \%$ for anxiety, $27.8 \%$ for insomnia, $38.9 \%$ for distress and $64.3 \%$ for compassion fatigue. In addition, women and younger people were at greater risk for symptoms. The data provide evidence for the need to establish prevention or intervention strategies to cope with mental health problems in health professionals.
\end{abstract}

Keywords

COVID-19; mental health; healthcare workers; anxiety; depression; distress; insomnia

\footnotetext{
${ }^{1}$ Correspondence about this article should be addressed to Alfonso Urzúa: alurzua@ucn.cl

${ }^{2}$ Escuela de Psicología, Universidad Católica del Norte. Avenida Angamos 0610, Antofagasta, Chile.
} 


\section{SYMPTOMATOLOGY ASSOCIATED WITH MENTAL HEALTH DISORDERS IN HEALTH WORKERS IN PARAGUAY: COVID-19 EFFECT}

\section{Introducción}

La enfermedad por COVID-19 (por sus siglas en inglés, Corona Virus Disease) es producida por el tipo de coronavirus SARS-CoV-2, la que se manifestó como brote infeccioso en Wuhan (China) en diciembre del año 2019 y tuvo una rápida propagación mundial, siendo Tailandia el lugar donde se confirmó el primer caso fuera del territorio chino. El 11 de marzo de 2020, la OMS declaró a la COVID-19 como pandemia debido a los elevados niveles de propagación y gravedad, así como por los niveles alarmantes de inacción ante este brote (OPS, 2020). En esta fecha, el epicentro ya se trasladaba a Europa (Italia y España, principalmente) y en el mundo se registraban más de 118000 casos confirmados y 4291 muertes por COVID-19 (OMS, 2020b). Por otra parte, en dicha fecha en Paraguay recién se habían confirmado cinco casos, ante lo cual el Ministerio de Salud Pública y Bienestar Social (MSPBS) y la Dirección General de Migraciones iniciaron estrictos controles a viajeros, se suspendieron los eventos públicos y privados de concurrencia masiva, así como las actividades educativas en todos los niveles (Ministerio de Salud Pública y Bienestar Social, 2020a).

Si bien la mayoría de los afectados por la enfermedad evolucionan favorablemente con recuperación sin necesidad de asistencia hospitalaria, 1 de cada 5 personas con COVID-19 pueden presentar una afección grave, sobre todo si se trata de personas mayores o si se presenta una patología de base. Fiebre, tos, disnea, opresión precordial y dificultades para hablar o moverse, son síntomas que requieren atención médica inmediata y lo recomendado es acudir a un profesional de la salud (Harapan et al, 2020; OMS, 2020a). Estos cuadros y aquellos que requieran hospitalización o asistencia respiratoria pueden derivar en el colapso del sistema sanitario, y por ende, al estrés al que estarían sometidos los profesionales de la salud, preocupación que en Paraguay se exacerbaba aún más cuando a inicios de marzo iniciaba el brote de COVID-19 y desde la dirección de Terapias del Ministerio de Salud se anunciaba que las terapias de Paraguay no darían abasto, así como tampoco la cantidad de personal de blanco capacitado para la atención (La Nación, 2020).

El 1 de mayo de 2020, cuando en el mundo existían 3.34 millones de casos confirmados y 238500 muertes por COVID-19, en Paraguay existían 333 casos confirmados, 10 muertes y 115 recuperados (208 casos activos). En esta fecha, durante la 
situación de emergencia establecida en Paraguay, y ante la amenaza de propagación de la COVID-19 en el país, los profesionales de la salud realizaron una manifestación en la que, además de equipos de bioseguridad y otros reclamos, exigieron justicia en la carga horaria laboral (El Trece, 2020). Según Wiskow y Hopfe (2020) del Departamento de Políticas Sectoriales de la OIT, ante el brote de la COVID-19 el personal de salud está expuesto a horas de trabajo prolongadas con una gran carga laboral adicional y sin contar con periodos adecuados de descanso. Las largas horas de trabajo, el exceso de atención diaria de pacientes, las posibles muertes de estos, así como tener que certificar la defunción o una enfermedad son factores predisponentes para desarrollar fatiga por compasión (O’Mahony et al., 2017).

La fatiga por compasión surgió en el campo de la enfermería (Moreno-Jiménez et al., 2004), es conocida con otros términos como desgaste por empatía, traumatización vicaria o estrés traumático secundario que surge como consecuencia de una relación empática y sensible con otras personas que están expuestas a trauma, tensión o estrés (González, González, Sánchez y Peña, 2018) y consiste en “el residuo emocional resultante de la exposición al trabajo con aquellos que sufren las consecuencias de eventos traumáticos" (Acinas, 2012, p. 8).

La sintomatología que deriva de la fatiga por compasión es muy diversa tanto en formas como en intensidad y duración, caracterizándose principalmente por síntomas que podrían presentarse repentinamente como reexperimentación de un evento traumático, evitación (y embotamiento psíquico) e hiperactividad, siendo naturales en situaciones que producen estrés (Acinas, 2012). Es decir, este constructo se basa en el hecho de que el origen puede ser por la experimentación de forma directa o indirecta (de otros), lo cual corresponde a uno de los criterios diagnósticos del Trastorno de Estrés post Traumático (TEPT) en el Manual Diagnóstico y Estadístico de los Trastornos Mentales (DSM) de la Asociación Americana de Psiquiatría (Beck, 2011).

El escenario en el cual se desempeñan los trabajadores de la salud también puede ser visto como estresante por encontrarse en continuo contacto con personas que sufren debido a diversos problemas (Gómez-Martínez et al., 2015). La calidad de vida y el desempeño socio-ocupacional pueden verse afectados negativamente por la ansiedad y la depresión acompañante (Rojtenberg, 2001; Yerkes y Dodson, 1908; Vera-Villarroel, 2020).

Existen varios estudios que confirman la presencia de ansiedad y depresión en trabajadores de la salud como los de medicina y enfermería. Betancourt et al. (2006) 
analizaron los síntomas de depresión y ansiedad en 90 residentes de medicina por medio de las escalas de Hamilton separándolos en dos grupos: 45 expuestos a estrés bajo (grupo control) y 45 expuestos a estrés alto (grupo problema), presentándose síntomas de depresión en el $44 \%$ y síntomas de ansiedad en el $43 \%$ de los participantes, concluyéndose que el estrés es el detonante de estos síntomas cuando se encuentran sometidos a estrés alto. Por otro lado, en la investigación de Erdur et al. (2006) participaron 192 médicos que trabajan en unidades de emergencia en Denizli (Turquía) de los cuales el 14.6\% presentó síntomas de ansiedad y el 15.1\% presentó síntomas de depresión, y en el estudio llevado a cabo por Cooke et al. (2007) encontraron que el 54\% del personal de enfermería que participó de su investigación presentó síntomas de ansiedad moderada o grave.

Los trabajadores de la salud expuestos a la enfermedad por el virus de la COVID19 pueden encontrarse estresados psicológicamente. Por esta razón, Lai et al. (2020) realizaron un estudio en el que participaron 1257 trabajadores de la salud de China, de los cuales 760 fueron de Wuhan, el epicentro de COVID-19 en ese país. El 71.5\% presentó síntomas de distrés, el $44.6 \%$ síntomas de ansiedad, 50.4\% síntomas de depresión y el 34\% síntomas de insomnio, presentándose con mayor severidad en el personal de enfermería, las mujeres, los profesionales de primera línea y quienes trabajaban en el epicentro del brote por COVID-19 (Wuhan).

Siguiendo esa misma línea, dada la importancia de determinar prevalencias de depresión, ansiedad, insomnio, distrés y fatiga por compasión, así como factores relacionados a la presencia de sintomatología con el fin de poder establecer estrategias de prevención o intervención basadas en la evidencia, se realizó el presente estudio exploratorio en profesionales de la salud de Paraguay.

\section{Método}

\section{Participantes}

Bajo un diseño transversal, se obtuvieron datos por medio de un formulario on line de 126 profesionales de salud, con edades entre 18 a 61 años $(M=32.22 ; D E=8.233)$ entre los que se encontraron profesionales de medicina, enfermería, psicología y de otras profesiones como kinesiología, odontología, auxiliares de enfermería, entre otros, todos ellos trabajando en establecimientos de salud de Paraguay. La selección se realizó por muestreo no probabilístico de tipo bola de nieve desde el 4 al 28 de abril de 2020. 
Del total de participantes, el $82.5 \%$ correspondió a mujeres, el $86.5 \%$ a mayores de 40 años, el $59.5 \%$ a solteros y el $57.9 \%$ a profesionales de la salud de medicina y enfermería Se les consultó si atendieron algún caso de infección respiratoria y de casos confirmados de COVID-19, a lo que el $47.6 \%$ respondió que atendió algún caso de infecciones respiratorias y el solo el $10.3 \%$ atendió algún caso confirmado de COVID19. De los 126 participantes del presente estudio, el $90.5 \%$ manifestó que contaba con protección individual (Tabla 1).

Tabla 1

Características sociodemográficas de la muestra

\begin{tabular}{llcc}
\hline & & $f$ & $\%$ \\
\hline Sexo & Hombres & 22 & 17.5 \\
Edad & Mujeres & 104 & 82.5 \\
& $18-25$ & 19 & 15.1 \\
& $26-30$ & 53 & 42.1 \\
& $31-40$ & 37 & 29.4 \\
Estado relacional & $>40$ & 17 & 13.5 \\
& Solteros $^{\mathrm{a}}$ & 78 & 61.9 \\
Ocupación & Casados $^{\mathrm{b}}$ & 48 & 38.1 \\
& Medicina & 44 & 34.9 \\
& Enfermería & 29 & 23.0 \\
Atención de infecciones respiratorias & Otros & 53 & 42.1 \\
& No & 66 & 52.4 \\
Atención de casos COVID-19 & Sí & 60 & 47.6 \\
& No & 113 & 89.7 \\
Protección individual & Sí & 13 & 10.3 \\
& No & 12 & 9.5 \\
& Sí & 114 & 90.5 \\
\hline
\end{tabular}

Nota ${ }^{\text {a }}$ La categoría casados incluyó a participantes convivientes $(\mathrm{n}=10)$; ${ }^{\mathrm{b}} \mathrm{La}$ categoría solteros incluyo a viudos $(\mathrm{n}=1)$ y divorciados $(\mathrm{n}=2)$

\section{Instrumentos}

\section{PHQ-9 (9-item Patient Health Questionnaire)}

Instrumento de 9 ítems que evalúa síntomas de depresión mediante cuatro alternativas de respuesta en formato Likert entre 0 a 3 puntos (ningún día, varios días, más de la mitad de los días y casi todos los días). Se obtiene un puntaje global equivalente a la sumatoria de las calificaciones de los 9 ítems que oscila entre 0 y 27 puntos. Presenta además un ítem adicional (PHQ_AD) que no se incorpora a la sumatoria total. La puntuación global puede categorizarse en cuatro niveles (normal: 0-4, leve: 5-9, moderado: 10-14 y severo: 15-21), así como también en dos categorías con punto de corte 
10 para depresión mayor. En el presente estudio se utilizó la versión en castellano (DiezQuevedo, Rangil, Sanchez-Planell, Kroenke y Spitzer, 2001) del original en inglés (Kroenke, Spitzer y Williams, 2001), obteniéndose un coeficiente de confiabilidad Omega de .884 con correlación ítem-total oscilando entre .494 y .739 , resultados que indican confiabilidad de las respuestas excelente sin necesidad de depurar ninguno de los ítems.

\section{GAD-7 (7-item Generalized Anxiety Disorder)}

Cuestionario de 7 ítems que evalúa síntomas de ansiedad a través de cuatro alternativas de respuesta para cada ítem en formato Likert calificados de 0 a 3 puntos (ningún día, varios días, más de la mitad de los días y casi todos los días). Se obtiene un puntaje global equivalente a la sumatoria de las calificaciones de los 7 ítems entre 0 y 21 puntos. La puntuación global puede categorizarse en cuatro niveles (normal: 0-4, leve: 59, moderado: 10-14 y severo: 15-21), así como también en dos categorías con punto de corte 7 para ansiedad. En el presente estudio se aplicó la versión en castellano (GarcíaCampayo et al., 2010) de la escala original (Spitzer, Kroenke, Williams y Löwe, 2006), la que obtuvo un coeficiente de confiabilidad Omega de .886 con correlación ítem-total oscilando entre .4936 y .787, resultados que indican confiabilidad de las respuestas excelente sin necesidad de depurar ninguno de los ítems.

ISI (7-item Insomnia Severity Index)

Instrumento que evalúa síntomas de insomnio a través de 7 ítems en formato de respuesta Likert de 5 alternativas: los tres primeros ítems (nada, leve, moderado, grave y muy grave); el ítem 4 (muy satisfecho, satisfecho, neutral, no muy satisfecho y muy satisfecho) y los tres últimos ítems (nada, un poco, algo, mucho y muchísimo). Las alternativas de respuesta se califican de 0 a 4 puntos, obteniéndose un puntaje global equivalente a la sumatoria de las calificaciones de los 7 ítems entre 0 y 28 puntos. La puntuación global puede categorizarse en cuatro niveles (normal: 0-7, subumbral o leve: 8-14, insomnio clínico de moderada severidad: 15-21 e insomnio clínico severo: 22-28), así como también en dos categorías con punto de corte 15 para insomnio. En el presente estudio se utilizó la versión en castellano (Sierra, Guillén-Serrano, Santos-Iglesias, 2008) de la escala original (Bastien, Vallieres y Morín, 2001), obteniéndose un coeficiente de confiabilidad Omega de .869 con correlación ítem-total oscilando entre 365 y .784, resultados que indican confiabilidad de las respuestas excelente sin necesidad de depurar ninguno de los ítems.

IES-R (22-item Impact of Event Scale-Revised) 
Cuestionario compuesto por 22 ítems que inquieren sobre síntomas de distrés, todos con cinco alternativas de respuesta en escala de tipo Likert (nada, un poco, algo, mucho y extremadamente) calificados de 0 a 4 puntos. Se obtiene un puntaje global equivalente a la sumatoria de las calificaciones de los 22 ítems el cual oscila entre 0 y 88 puntos. La puntuación global puede categorizarse en cuatro niveles (normal: 0-8, leve: 925, moderado: 26-43 y severo: 44-88), así como también en dos categorías con punto de corte 26 para distrés. En el presente estudio utilizó la versión en castellano (Báguena, Villarroya, Beleña et al., 2001), del original en inglés (Weiss, 1984), obteniéndose un coeficiente de confiabilidad Omega de .967 con correlación ítem-total oscilando entre .642 y .873 , resultados que indican confiabilidad de las respuestas excelente sin necesidad de depurar ninguno de los ítems.

Subescala de Fatiga por compasión del ProQOL - CSF-vIV (Professional Quality of Life: Compassion Satisfaction and Fatigue Subscales)

El instrumento ProQOL - CSF-vIV está compuesto por tres subescalas: Burnout, satisfacción por compasión y fatiga por compasión, teniendo un total de 30 ítems. En esta investigación se aplicó la subescala de Fatiga por compasión que evalúa dicho constructo por medio de 10 ítems con cinco alternativas de respuesta en formato Likert calificados de 0 a 5 puntos (nunca, raramente, algunas veces, con alguna frecuencia, casi siempre y siempre). Se obtiene un puntaje de fatiga por compasión equivalente a la sumatoria de las calificaciones de los 10 ítems, pudiendo oscilar entre 0 y 50 puntos. La puntuación obtenida puede categorizarse en cuatro niveles (normal: 0-7, leve: 8-13, moderado: 1417 y severo: 18-100). La puntación se basa en puntos de corte teóricos que derivan de varias investigaciones y se establece una media de 13, puntaje que se empleó para la categorización en dos niveles siendo el punto de corte 13 para fatiga por compasión (Stamm, 1997-2005). En el presente estudio se obtuvo un coeficiente de confiabilidad Omega de .803 con correlación ítem-total oscilando entre .271 y .641, resultados que indican confiabilidad de las respuestas adecuada sin necesidad de depurar ninguno de los ítems.

\section{Procedimiento}

Antes de comenzar a llenar el cuestionario online, se mostró a los participantes el objetivo general del proyecto de investigación y la importancia de este, con la finalidad que pudieran otorgar informadamente su consentimiento de manera online, 
garantizándoles su anonimato y la confidencialidad de los datos. Se les informó que podrían retirarse en cualquier momento de la evaluación y se les brindó un dato de contacto en caso de desear realizar alguna consulta. La investigación no conllevó ningún tipo de riesgo para los participantes.

Por medio de un cuestionario sociodemográfico se consultó el sexo, la edad, el estado civil, la ocupación actual y si atendió casos de infecciones respiratorias y confirmados de COVID-19. Así también, se preguntó si contaban con protección personal.

Los datos rescatados en planilla Excel, fueron transformados de formato para ser analizados en el programa SPSS 25.0 (IBM, 2017). Se calculó la frecuencia de participantes por cada subgrupo de las variables sociodemográficas estudiadas, así como el porcentaje correspondiente.

Las puntuaciones obtenidas en depresión, ansiedad, insomnio, distrés y fatiga por compasión se categorizaron en cuatro niveles (normal, leve, moderado y severo), de acuerdo a los puntos de corte descritos en la sección de instrumentos. Las proporciones de severidad de los niveles se compararon por medio de la prueba Chi cuadrado para proporciones.

Para comparar las puntuaciones de los subgrupos, se verificó la distribución de las mismas por medio de la prueba de Kolmogorov-Smirnov (para subgrupos con más de 50 participantes) y de la prueba de Shapiro Wilk (para subgrupos con menos de 50 participantes). Como no se verificó distribución normal, se optó por la prueba U de Mann Whitney para la comparación entre dos grupos y la prueba de Spearman para la correlación entre las puntuaciones totales. Para determinar los subgrupos con mayor puntuación en cada variable, se calculó la mediana y el rango intercuartil.

Por último, se realizó un análisis de regresión multinomial para calcular los factores de riesgo (ORs) y el 95\% del intervalo de confianza correspondiente.

\section{Resultados}

\section{Gravedad de la sintomatología}

Las tablas 2 y 3 nos muestran los porcentajes de participantes por categorías de severidad de la sintomatología reportada por sexo, ocupación, atención de casos y uso de protección personal. Para el total de la muestra, los porcentajes de participantes que reportaron síntomas clasificables como moderados y severos fueron de un $32.2 \%$ para 
depresión, $41.3 \%$ en ansiedad, $27.8 \%$ en insomnio, $38.9 \%$ en distrés y un $64.3 \%$ en fatiga por compasión.

Las mujeres presentaron mayor proporción de síntomas severos de depresión ( $p=$ $.023)$, ansiedad $(p=.035)$, insomnio $(p=.024)$, distrés $(p=.001)$ y fatiga por compasión ( $p=.048)$ (Tabla 2). Así también, los síntomas de insomnio (moderada y severa) se presentó en mayor proporción en quienes no atendieron casos de infecciones respiratorias $(p=.014)$ y la fatiga por compasión severa fue mayor en los profesionales de medicina $(p=.01)$.

\section{Tabla 2}

Categorías de gravedad de las mediciones de depresión, ansiedad, insomnio, distrés y fatiga por sexo y ocupación

\begin{tabular}{|c|c|c|c|c|c|c|c|c|c|}
\hline & \multirow{3}{*}{$\begin{array}{c}\text { Categoría } \\
\text { de } \\
\text { severidad }\end{array}$} & \multirow{3}{*}{$\begin{array}{l}\text { Total } \\
f(\%)\end{array}$} & \multicolumn{3}{|c|}{ Sexo } & \multicolumn{4}{|c|}{ Ocupación } \\
\hline & & & $f(\%)$ & $f(\%)$ & & $f(\%)$ & $f(\%)$ & & \\
\hline & & & Hombres & Mujeres & $p$ & Medicina & Enfermería & Otros & $P$ \\
\hline \multirow{4}{*}{ Depresión } & Normal & $43(34.1)$ & $13(59.1)$ & $30(28.8)$ & \multirow{4}{*}{.023} & $12(27.3)$ & $8(27.6)$ & $23(43.4)$ & \multirow{4}{*}{.280} \\
\hline & Leve & $42(33.3)$ & $7(31.8)$ & $35(33.7)$ & & $16(36.4)$ & $12(41.4)$ & $14(26.4)$ & \\
\hline & Moderada & $28(22.2)$ & $1(4.5)$ & $27(26)$ & & $11(25)$ & $4(13.8)$ & $13(24.5)$ & \\
\hline & Severa & 13(10.3) & $1(4.5)$ & $12(11.5)$ & & $5(11.4)$ & $5(17.2)$ & $3(5.7)$ & \\
\hline \multirow{4}{*}{ Ansiedad } & Normal & $29(23)$ & $9(40.9)$ & $20(19.2)$ & \multirow{4}{*}{.035} & $10(22.7)$ & $3(10.3)$ & $16(30.2)$ & \multirow{4}{*}{$.31 c$} \\
\hline & Leve & $45(35.7)$ & $9(40.9)$ & $36(34.6)$ & & $18(40.9)$ & $9(31)$ & $18(34)$ & \\
\hline & Moderada & $33(26.2)$ & $4(18.2)$ & $29(27.9)$ & & $9(20.5)$ & $11(37.9)$ & $13(24.5)$ & \\
\hline & Severa & 19(15.1) & $0(0)$ & 19(18.3) & & $7(15.9)$ & $6(20.7)$ & $6(11.3)$ & \\
\hline \multirow{4}{*}{ Insomnio } & Normal & $40(31.7)$ & $13(59.1)$ & $27(26)$ & \multirow{4}{*}{.024} & $13(29.5)$ & $8(27.6)$ & $19(35.8)$ & \multirow{4}{*}{.244} \\
\hline & Leve & $51(40.5)$ & $6(27.3)$ & $45(43.3)$ & & $21(47.7)$ & $14(48.3)$ & $16(30.2)$ & \\
\hline & Moderado & $34(27)$ & $3(13.6)$ & $31(29.8)$ & & $10(22.7)$ & $6(20.7)$ & $18(34)$ & \\
\hline & Severo & $1(0.8)$ & $0(0)$ & $1(1)$ & & $0(0)$ & $1(3.4)$ & $0(0)$ & \\
\hline \multirow{4}{*}{ Distrés } & Normal & $30(23.8)$ & $11(50)$ & 19(18.3) & \multirow{4}{*}{.001} & $13(29.5)$ & $5(17.2)$ & $12(22.6)$ & \multirow{4}{*}{.313} \\
\hline & Leve & $47(37.3)$ & $10(45.5)$ & $37(35.6)$ & & $16(36.4)$ & $8(27.6)$ & $23(43.4)$ & \\
\hline & Moderada & $29(23)$ & $1(4.5)$ & $28(26.9)$ & & $8(18.2)$ & $8(27.6)$ & $13(24.5)$ & \\
\hline & Severa & 20(15.9) & $0(0)$ & $20(19.2)$ & & 7(15.9) & $8(27.6)$ & $5(9.4)$ & \\
\hline \multirow{4}{*}{$\begin{array}{l}\text { Fatiga por } \\
\text { compasión }\end{array}$} & Normal & $18(14.3)$ & $3(13.6)$ & $15(14.4)$ & \multirow{4}{*}{.048} & $2(4.5)$ & $2(6.9)$ & $14(26.4)$ & \multirow{4}{*}{.011} \\
\hline & Leve & $27(21.4)$ & $8(36.4)$ & 19(18.3) & & $14(31.8)$ & $4(13.8)$ & $9(17)$ & \\
\hline & Moderada & $27(21.4)$ & $7(31.8)$ & $20(19.2)$ & & $8(18.2)$ & $6(20.7)$ & $13(24.5)$ & \\
\hline & Severa & $54(42.9)$ & $4(18.2)$ & $50(48.1)$ & & $20(45.5)$ & $17(58.6)$ & $17(32.1)$ & \\
\hline
\end{tabular}


Tabla 3

Categorías de gravedad de las mediciones de depresión, ansiedad, insomnio, distrés y fatiga por compasión en los subgrupos

\begin{tabular}{|c|c|c|c|c|c|c|c|c|c|c|c|}
\hline & \multirow{3}{*}{$\begin{array}{c}\text { Categoría } \\
\text { de } \\
\text { severidad }\end{array}$} & \multirow{3}{*}{$\begin{array}{l}\text { Total } \\
f(\%)\end{array}$} & \multicolumn{3}{|c|}{$\begin{array}{l}\text { Atención de infecciones } \\
\text { respiratorias }\end{array}$} & \multicolumn{3}{|c|}{$\begin{array}{l}\text { Atención de casos } \\
\text { COVID-19 }\end{array}$} & \multicolumn{3}{|c|}{ Protección individual } \\
\hline & & & $f(\%)$ & $f(\%)$ & & $f(\%)$ & $f(\%)$ & & $f(\%)$ & $f(\%)$ & \\
\hline & & & No & Sí & $p$ & No & Sí & $p$ & No & Sí & $P$ \\
\hline \multirow{4}{*}{ Depresión } & Normal & $43(34.1)$ & $27(40.9)$ & $16(26.7)$ & \multirow{4}{*}{.135} & $40(35.4)$ & $3(23.1)$ & \multirow{4}{*}{.721} & $4(33.3)$ & $39(34.2)$ & \multirow{4}{*}{.872} \\
\hline & Leve & $42(33.3)$ & $16(24.2)$ & $26(43.3)$ & & $36(31.9)$ & $6(46.2)$ & & $4(33.3)$ & $38(33.3)$ & \\
\hline & Moderada & $28(22.2)$ & $16(24.2)$ & $12(20)$ & & $25(22.1)$ & $3(23.1)$ & & 2(16.7) & $26(22.8)$ & \\
\hline & Severa & $13(10.3)$ & $7(10.6)$ & $6(10)$ & & $12(10.6)$ & $1(7.7)$ & & $2(16.7)$ & $11(9.6)$ & \\
\hline \multirow{4}{*}{ Ansiedad } & Normal & $29(23)$ & $19(28.8)$ & $10(16.7)$ & \multirow{4}{*}{.220} & $26(23)$ & $3(23.1)$ & \multirow{4}{*}{.274} & $2(16.7)$ & $27(23.7)$ & \multirow{4}{*}{.942} \\
\hline & Leve & $45(35.7)$ & $19(28.8)$ & $26(43.3)$ & & $43(38.1)$ & $2(15.4)$ & & $5(41.7)$ & $40(35.1)$ & \\
\hline & Moderada & $33(26.2)$ & $19(28.8)$ & $14(23.3)$ & & $27(23.9)$ & $6(46.2)$ & & $3(25)$ & $30(26.3)$ & \\
\hline & Severa & 19(15.1) & $9(13.6)$ & $10(16.7)$ & & $17(15)$ & $2(15.4)$ & & $2(16.7)$ & $17(14.9)$ & \\
\hline \multirow{4}{*}{ Insomnio } & Normal & $40(31.7)$ & $26(39.4)$ & $14(23.3)$ & \multirow{4}{*}{.014} & $36(31.9)$ & $4(30.8)$ & \multirow{4}{*}{.762} & $3(25)$ & $37(32.5)$ & \multirow{4}{*}{.601} \\
\hline & Leve & $51(40.5)$ & $18(27.3)$ & $33(55)$ & & $47(41.6)$ & $4(30.8)$ & & $7(58.3)$ & 44(38.6) & \\
\hline & Moderado & $34(27)$ & $21(31.8)$ & $13(21.7)$ & & $29(25.7)$ & $5(38.5)$ & & $2(16.7)$ & $32(28.1)$ & \\
\hline & Severo & $1(0.8)$ & $1(1.5)$ & $0(0)$ & & $1(0.9)$ & $0(0)$ & & $0(0)$ & $1(0.9)$ & \\
\hline \multirow{4}{*}{ Distrés } & Normal & $30(23.8)$ & $19(28.8)$ & $11(18.3)$ & \multirow{4}{*}{.454} & $28(24.8)$ & $2(15.4)$ & \multirow{4}{*}{.106} & $3(25)$ & $27(23.7)$ & \multirow{4}{*}{.903} \\
\hline & Leve & $47(37.3)$ & $21(31.8)$ & 26(43.3) & & $42(37.2)$ & $5(38.5)$ & & $5(41.7)$ & $42(36.8)$ & \\
\hline & Moderada & $29(23)$ & $15(22.7)$ & $14(23.3)$ & & $23(20.4)$ & $6(46.2)$ & & $3(25)$ & $26(22.8)$ & \\
\hline & Severa & $20(15.9)$ & $11(16.7)$ & $9(15)$ & & $20(17.7)$ & $0(0)$ & & $1(8.3)$ & 19(16.7) & \\
\hline \multirow{4}{*}{$\begin{array}{l}\text { Fatiga por } \\
\text { compasión }\end{array}$} & Normal & $18(14.3)$ & 13(19.7) & $5(8.3)$ & \multirow{4}{*}{.341} & $17(15)$ & $1(7.7)$ & \multirow{4}{*}{.150} & $0(0)$ & $18(15.8)$ & \multirow{4}{*}{.130} \\
\hline & Leve & $27(21.4)$ & 13(19.7) & $14(23.3)$ & & $25(22.1)$ & $2(15.4)$ & & $5(41.7)$ & $22(19.3)$ & \\
\hline & Moderada & $27(21.4)$ & 13(19.7) & $14(23.3)$ & & 21(18.6) & $6(46.2)$ & & $1(8.3)$ & $26(22.8)$ & \\
\hline & Severa & $54(42.9)$ & $27(40.9)$ & $27(45)$ & & $50(44.2)$ & $4(30.8)$ & & $6(50)$ & $48(42.1)$ & \\
\hline
\end{tabular}

\section{Sintomatología total según subgrupos}

Las mujeres presentaron puntajes significativamente más altos que los hombres en depresión $(p=.001)$, ansiedad $(p=.004)$, insomnio $(p=.001)$, distrés $(p=.000)$ y fatiga por compasión ( $p=.044$ ) (Tabla 4). La ansiedad fue significativamente mayor en los profesionales de enfermería en comparación con los demás profesionales de la salud ( $p=.038)$. La fatiga por compasión fue significativamente mayor en los profesionales de enfermería $(p=.004)$ y los de medicina $(p=.022)$ al compararlos con los demás profesionales de la salud que participaron de la investigación (Tabla 4). 
Tabla 4

Puntuaciones de las mediciones de depresión, ansiedad, insomnio, distrés y fatiga por compasión por sexo y ocupación

$$
\text { Sexo }
$$

Ocupación

Mediana (IQR)

Mediana (IQR)

\begin{tabular}{lccccccccc} 
& Hombres & Mujeres & $p$ & Medicina & Enfermería & Otros & $p^{a}$ & $p^{b}$ & $p^{c}$ \\
\hline \multirow{2}{*}{ Depresión } & 4 & 8 & .001 & 8 & 8 & 6 & .919 & .454 & .445 \\
& $(2-5)$ & $(4-12)$ & & $(4-10)$ & $(4-10)$ & $(3-11)$ & & & \\
\hline \multirow{2}{*}{ Ansiedad } & 6 & 9 & .004 & 8 & 11 & 7 & .229 & .441 & .038 \\
& $(2-9)$ & $(6-13.5)$ & & $(5-12)$ & $(7-14)$ & $(4-11)$ & & & \\
\hline \multirow{2}{*}{ Insomnio } & 5 & 12 & .001 & 11 & 10 & 11 & .709 & .762 & .931 \\
& $(3-10)$ & $(7-16)$ & & $(6.5-14)$ & $(7-14)$ & $(5-16)$ & & & \\
\hline \multirow{2}{*}{ Distrés } & 9 & 25 & .000 & 21 & 29 & 19 & .223 & .698 & .087 \\
& $(3-19)$ & $(14-41)$ & & $(7.5-31)$ & $(16-46)$ & $(9-33)$ & & & \\
\hline Fatiga por & 12 & 16 & .044 & 15 & 18 & 13 & .480 & .022 & .004 \\
compasión & $(9-15)$ & $(11-21)$ & & $(11-24)$ & $(13-24)$ & $(7-18)$ & & & \\
\hline
\end{tabular}

Nota. ${ }^{\text {a }}$ Medicina versus Enfermería; ${ }^{\mathrm{b}}$ Medicina versus otros; ${ }^{\mathrm{c}}$ Enfermería versus otros

No se encontraron diferencias estadísticamente significativas dadas por la atención o no a pacientes con enfermedades respiratorias o con COVID-19 y por el uso de protección individual (Tabla 5).

Tabla 5

Puntuaciones de las mediciones de depresión, ansiedad, insomnio, distrés y fatiga por compasión por atención y uso de protección

\begin{tabular}{|c|c|c|c|c|c|c|c|c|c|}
\hline & $\begin{array}{l}\text { Atenció } \\
\text { respirat }\end{array}$ & $\begin{array}{l}\text { de infecciones } \\
\text { as }\end{array}$ & & $\begin{array}{l}\text { Atención } \\
\text { COVID- }\end{array}$ & e casos & & Protección & dividual & \\
\hline & Med & na (IQR) & & Medial & & & Mediana & & \\
\hline & No & Sí & $p$ & No & Sí & $p$ & No & Sí & $P$ \\
\hline & 6 & 8 & .575 & 7 & 8 & .763 & 6 & 7 & .937 \\
\hline Depiesionio & $(3-12)$ & $(4-10)$ & & $(4-11)$ & $(5-10)$ & & $(3.5-10)$ & $(4-11)$ & \\
\hline A pcisdod & 8 & 8 & .550 & 8 & 12 & .379 & 9 & 8 & .733 \\
\hline 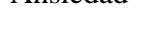 & $(4-13)$ & $(6-12)$ & & $(5-12)$ & $(8-13)$ & & $(6-12.5)$ & $(5-13)$ & \\
\hline Inson & 10 & 11 & .518 & 11 & 9 & .995 & 11 & 11 & .993 \\
\hline 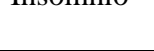 & $(5-16)$ & $(8-14)$ & & $(6-15)$ & $(7-16)$ & & $(7.5-13.5)$ & $(6-15)$ & \\
\hline Distrác & 20 & 21 & .665 & 20 & 21 & .984 & 22 & 21 & .884 \\
\hline 年 & $(8-38)$ & $(12-34.5)$ & & $(9-38)$ & $(16-29)$ & & $(12-35)$ & $(9-37)$ & \\
\hline Fatiga por & 15 & 15 & .206 & 15 & 14 & .819 & 20 & 15 & .160 \\
\hline compasi & $(9-19)$ & $(11.5-22.5)$ & & $(10-21)$ & $(13-17)$ & & $(11.5-27)$ & $(10-20)$ & \\
\hline
\end{tabular}




\section{Relación de la sintomatología con otras variables}

En cuanto a la correlación de las variables principales con la edad, la probabilidad de contagio propio, la probabilidad que otros se contagien y los días desde el primer caso confirmado de COVID-19 (Tabla 6), se verificó correlación estadísticamente significativa y negativa de la edad con la depresión $\left(r_{S(126)}=.253 ; p=.004\right)$, la ansiedad $\left(r_{S(126)}=.228\right.$; $p=.010)$ y el distrés $\left(r_{S(126)}=.175 ; p=.0497\right)$. Estos resultados indican una relación inversa, es decir, a menor edad se presentaría mayor depresión, mayor ansiedad y mayor distrés.

Tabla 6

Correlaciones de las mediciones de salud mental con la edad, la probabilidad de contagio propio, la probabilidad de que otros se contagien y la cantidad de días desde el primer caso confirmado

\begin{tabular}{lccccccccccc}
\hline & \multicolumn{2}{c}{ Depresión } & \multicolumn{2}{c}{ Ansiedad } & \multicolumn{2}{c}{ Insomnio } & \multicolumn{2}{c}{ Distrés } & \multicolumn{3}{c}{$\begin{array}{c}\text { Fatiga por } \\
\text { compasión }\end{array}$} \\
& \multicolumn{1}{c}{$r_{S}$} & $p$ & $r_{S}$ & $p$ & $r_{S}$ & $p$ & $r_{S}$ & $P$ & $r_{S}$ & $p$ \\
\hline Edad & $-.253^{* *}$ & .004 & $-.228^{*}$ & .010 & -.097 & .280 & - & $.175^{*}$ & .0497 & -.0795 & .376 \\
$\begin{array}{l}\text { Probabilidad de } \\
\text { contagio propio }\end{array}$ & .131 & .143 & .162 & .070 & .030 & .736 & .024 & .790 & .125 & .164 \\
$\begin{array}{l}\text { Probabilidad de que } \\
\text { otros se contagien }\end{array}$ & .108 & .227 & .098 & .274 & .122 & .175 & .056 & .530 & .064 & .477 \\
$\begin{array}{l}\text { Días desde el primer } \\
\text { caso confirmado }\end{array}$ & .108 & .229 & .008 & .926 & .017 & .854 & -.110 & .221 & .021 & .812 \\
\hline
\end{tabular}

Nota. $* p<.05 ; * * p<.01$

\section{Riesgo}

El OR fue calculado para cada variable utilizando como categorías sexo, ocupación, estado civil, atención y uso de protección (Tabla 7). Las mujeres manifestaron mayor riesgo de manifestar depresión $(p=.007)$, de ansiedad $(p=.013)$, distrés $(p=.005)$ y fatiga por compasión $(p=.014)$. Por otra parte, la fatiga por compasión también se presenta con mayor riesgo en los profesionales de enfermería $(p=.0025)$ y en los solteros $(p=.039)$.

\section{Tabla 7}

Factores de riesgo identificados por análisis de regresión logística multivariable

\begin{tabular}{lllll}
\hline Variables & \multicolumn{2}{l}{ Casos severos/ Total $(\%)$} & OR (95\%IC) & $P$ \\
\hline PHQ-9 & & & & \\
Sexo & Hombres & $2 / 22(9.1)$ & Referencia & \\
& Mujeres & $39 / 104(37.5)$ & $9.3(1.9-47.1)^{* *}$ & .007 \\
Ocupación & Otros & $16 / 44(36.4)$ & Referencia & \\
& Medicina & $9 / 29(31)$ & $2.5(0.9-7.3)$ & .081 \\
Estado civil & Enfermería & $16 / 53(30.2)$ & $1(0.3-2.9)$ & .991 \\
Atención de infecciones & Casados & $17 / 51(33.3)$ & Referencia & .808 \\
respiratorias & Solteros & $24 / 75(32)$ & $1.1(0.5-2.5)$ & .593 \\
Atención de casos COVID-19 & No & $23 / 66(34.8)$ & Referencia & $.59(0.3-2)$ \\
\end{tabular}




\begin{tabular}{|c|c|c|c|c|}
\hline Protección individual & $\begin{array}{l}\text { Sí } \\
\text { Sí } \\
\text { No }\end{array}$ & $\begin{array}{l}4 / 13(30.8) \\
37 / 114(32.5) \\
4 / 12(33.3)\end{array}$ & $\begin{array}{l}0.9(0.2-3.7) \\
\text { Referencia } \\
0.7(0.2-2.9)\end{array}$ & .868 \\
\hline \multicolumn{5}{|l|}{ GAD-7 } \\
\hline \multirow{3}{*}{ Sexo } & Hombres & $10 / 22(45.5)$ & Referencia & \\
\hline & Mujeres & 73/104(70.2) & $4.1(1.3-12.4)^{* *}$ & .013 \\
\hline & Otros & $30 / 44(68.2)$ & Referencia & \\
\hline \multirow[t]{2}{*}{ Ocupación } & Medicina & $22 / 29(75.9)$ & $1.8(0.7-5.1)$ & .255 \\
\hline & Enfermería & $31 / 53(58.5)$ & $1.5(0.5-4.5)$ & .443 \\
\hline \multirow{2}{*}{ Estado civil } & Casados & $31 / 51(60.8)$ & Referencia & \\
\hline & Solteros & $52 / 75(69.3)$ & $1.7(0.8-3.8)$ & .191 \\
\hline \multirow{2}{*}{$\begin{array}{l}\text { Atención de infecciones } \\
\text { respiratorias }\end{array}$} & No & $40 / 66(60.6)$ & Referencia & \\
\hline & Sí & $43 / 60(71.7)$ & $1.6(0.6-4.1)$ & .319 \\
\hline \multirow{2}{*}{ Atención de casos COVID-19 } & No & $73 / 113(64.6)$ & Referencia & \\
\hline & Sí & $10 / 13(76.9)$ & $1.2(0.3-5.4)$ & .822 \\
\hline \multirow{2}{*}{ Protección individual } & Sí & 74/114(64.9) & Referencia & \\
\hline & No & $9 / 12(75)$ & $1.7(0.4-7.6)$ & .505 \\
\hline \multicolumn{5}{|l|}{ ISI } \\
\hline \multirow{3}{*}{ Sexo } & Hombres & $3 / 22(13.6)$ & Referencia & \\
\hline & Mujeres & $32 / 104(30.8)$ & $3.1(0.8-12.3)$ & .113 \\
\hline & Otros & $10 / 44(22.7)$ & Referencia & \\
\hline \multirow[t]{2}{*}{ Ocupación } & Medicina & $7 / 29(24.1)$ & $0.9(0.3-2.7)$ & .922 \\
\hline & Enfermería & $18 / 53(34)$ & $0.6(0.2-1.8)$ & .344 \\
\hline \multirow{2}{*}{ Estado civil } & Casados & $11 / 51(21.6)$ & Referencia & \\
\hline & Solteros & $24 / 75(32)$ & $1.8(0.7-4.2)$ & .196 \\
\hline \multirow{2}{*}{$\begin{array}{l}\text { Atención de infecciones } \\
\text { respiratorias }\end{array}$} & No & $22 / 66(33.3)$ & Referencia & \\
\hline & Sí & $13 / 60(21.7)$ & $0.5(0.2-1.5)$ & .230 \\
\hline \multirow{2}{*}{ Atención de casos COVID-19 } & No & $30 / 113(26.5)$ & Referencia & \\
\hline & Sí & $5 / 13(38.5)$ & $2.5(0.6-9.9)$ & .206 \\
\hline \multirow{2}{*}{ Protección individual } & Sí & $33 / 114(28.9)$ & Referencia & \\
\hline & No & 2/12(16.7) & $0.6(0.1-3)$ & .506 \\
\hline \multicolumn{5}{|l|}{ IES-R } \\
\hline \multirow{3}{*}{ Sexo } & Hombres & $1 / 22(4.5)$ & Referencia & \\
\hline & Mujeres & $48 / 104(46.2)$ & $20.7(2.5-173.4)^{* *}$ & .005 \\
\hline & Otros & 15/44(34.1) & Referencia & \\
\hline \multirow[t]{2}{*}{ Ocupación } & Medicina & $16 / 29(55.2)$ & $1.9(0.7-5.5)$ & .218 \\
\hline & Enfermería & 18/53(34) & $2(0.7-5.5)$ & .173 \\
\hline Ectodo civil & Casados & $19 / 51(37.3)$ & Referencia & \\
\hline Estado civil & Solteros & $30 / 75(40)$ & $1.2(0.6-2.7)$ & .615 \\
\hline Atención de infecciones & No & 26/66(39.4) & Referencia & \\
\hline respiratorias & Sí & $23 / 60(38.3)$ & $0.9(0.4-2.2)$ & .815 \\
\hline Attrción de cocec COVID 10 & No & $43 / 113(38.1)$ & Referencia & \\
\hline Alention de casos Cuvite-19 & Sí & 6/13(46.2) & $1.1(0.3-4.5)$ & .860 \\
\hline & Sí & $45 / 114(39.5)$ & Referencia & \\
\hline Proteccion individual & No & $4 / 12(33.3)$ & $0.6(0.2-2.7)$ & .548 \\
\hline Fatiga por compasión & & & & \\
\hline Sexo & Hombres & $4 / 22(18.2)$ & Referencia & \\
\hline Sexo & Mujeres & $50 / 104(48.1)$ & $0.2(0.1-0.7)^{*}$ & .014 \\
\hline & Otros & $20 / 44(45.5)$ & Referencia & \\
\hline Ocupación & Medicina & $17 / 29(58.6)$ & $0.4(0.1-1)$ & .053 \\
\hline & Enfermería & $17 / 53(32.1)$ & $0.3(0.1-0.9)^{*}$ & .025 \\
\hline & Casados & 28/51(54.9) & Referencia & \\
\hline Estado civil & Solteros & $26 / 75(34.7)$ & $2.3(1-5.1) *$ & .039 \\
\hline Atención de infecciones & No & 27/66(40.9) & Referencia & \\
\hline respiratorias & Sí & $27 / 60(45)$ & $0.9(0.4-2.3)$ & .903 \\
\hline Atención de casos COVID-19 & No & $50 / 113(44.2)$ & Referencia & \\
\hline Atencion de casos CUVID-19 & Sí & $4 / 13(30.8)$ & $2.4(0.6-9.8)$ & .234 \\
\hline Protección individual & Sí & 48/114(42.1) & Referencia & \\
\hline Protection indiviaual & No & $6 / 12(50)$ & $1(0.3-3.8)$ & .987 \\
\hline
\end{tabular}




\section{Discusión}

Este estudio tuvo por objetivo el evaluar la prevalencia de sintomatología asociada a problemas de salud mental (ansiedad, depresión, insomnio, distrés y fatiga por compasión).

En términos generales, uno de cada tres participantes reportó síntomas moderados o severos de depresión, ansiedad, insomnio y distrés. Dos de cada tres evaluados reportó síntomas moderados o severos de fatiga por compasión. La presencia de sintomatología asociada a problemas de salud mental era esperable, dada las circunstancias de estrés y exigencias a las a que se ha visto sometido el equipo de salud, sin embargo, son menores a las reportadas utilizando similares instrumentos al estudio realizado en China (Lai, 2020), donde se encontró que más del $50 \%$ de los encuestados reportó síntomas de depresión y un $45 \%$ de ansiedad y en Chile, donde el $65 \%$ de la muestra reportó síntomas de depresión, 74\% de ansiedad, 65\% insomnio y 57\% de distrés (Urzúa, Vera-Villarroel, Samaniego et al., 2020). Existen diversas explicaciones y que podrían estar vinculadas por un lado a un riesgo percibido como alejado al momento de la recolección de datos en el mes de abril, considerando que la gran mayoría (un 90\%), aún no ha atendido a pacientes infectados, sumado al bajo número de casos en el país, hecho que origina, por ende, una menor demanda de los servicios críticos y de sobrecarga laboral. A esto se adiciona que el 90,5\% declaró usar siempre protección individual, lo que proporciona una sensación de mayor seguridad que incide en la presencia de síntomas, lo que se constituye en un factor protector como el uso de estrictos protocolos de seguridad (Khalid, Khalid, Qabajah, Barnard, y Qushmaq, 2016). No obstante, los resultados pudieron haberse incrementado dado que, en el periodo posterior a la recolección de los datos, los casos confirmados en Paraguay casi se han duplicado en un periodo de ocho días, aumentando de 266 que se tenía el 30 de abril hasta 563 casos confirmados el 8 de mayo de 2020 (MSPBS, 2020b), lo que sugiere la importancia de realizar un seguimiento de estos indicadores por mayor tiempo.

Al comparar los resultados estratificando por grupos, las mujeres son las que presentaron mayor proporción de síntomas severos y a la vez mayor riesgo de presentar depresión, ansiedad, distrés y fatiga por compasión. Las mujeres como grupo con mayor vulnerabilidad en los trabajadores de la salud ya han sido reportado en otros estudios en COVID-19 (Lai, 2020; Urzúa, Vera-Villarroel, Samaniego et al., 2020) y otros brotes similares como el SARS (Tam, Pang, Lam, y Chiu, 2004). No es posible abordar este 
aspecto sin entrar a discusiones de género e interseccionalidad que escapan a este manuscrito, pero asumimos que ambas perspectivas son capaces de explicar por qué el hecho de ser mujer constituye un mayor riesgo y genera una mayor vulnerabilidad, dado por una mayor morbilidad psiquiátrica, influencia de roles sociales, influencia del ciclo reproductivo, entre otros (Montero et al., 2004).

Los resultados también mostraron una relación con la edad, los cuales muestran que a menor edad mayor cantidad de sintomatología en salud mental. Esta tendencia encontrada entrega información relevante acerca de las competencias socioemocionales de las nuevas generaciones de profesionales de la salud que deben ser fortalecidas o desarrolladas más allá de las competencias técnicas específicas de la disciplina en particular. Es importante dilucidar en nuevos estudios si esto es particularmente importante solo entre los profesionales de Paraguay o es más bien una tendencia que trasciende otros países y/o culturas ya que similar hallazgo se encontró en el estudio chileno (Urzúa, Vera-Villarroel, Samaniego, Caqueo-Urízar, Zapata, y Irarrázaval, 2020).

El presente estudio tiene limitantes que pueden ser asumidas en estudios posteriores. En primer lugar, dado el carácter transversal del diseño, no fue posible contar con una línea base de las variables de salud mental evaluadas, por lo tanto, aunque asumimos que el COVID-19 tiene un efecto directo sobre el aumento de la sintomatología, al no ser un estudio longitudinal no podemos realizar relaciones de tipo causal, sino solo asociaciones que pueden orientar la focalización de grupos más vulnerables en tanto riesgo o mayor presencia de síntomas. Una segunda limitante tiene que ver con el tamaño muestral y al tipo de muestreo, dado que no es una muestra representativa del universo de trabajadores de la salud del Paraguay, sino que, dado lo complejo del contexto sanitario, corresponde a una muestra obtenida a través de redes y utilizando el muestreo de bola de nieve, que aun cuando es usado con estos fines en poblaciones de difícil acceso, limita la extrapolación de los resultados al universo muestral.

Sería interesante poder evaluar la evolución de la salud mental a medida que aumentan los casos, especialmente en el personal de salud, así como posibles diferencias dadas en los sectores público o privado o en los distintos niveles de atención en salud (primaria y secundaria).

Pese a esto, creemos que los resultados obtenidos en este primer acercamiento constituyen un aporte a la evidencia que permite apoyar estrategias de intervención en los equipos de salud desde la perspectiva psicológica (Urzúa, Vera-Villarroel, Caqueo-Urízar 
y Polanco, 2020), ya que frecuentemente estos no son considerados en las políticas públicas como sujetos de intervención, aun cuando sabemos que no solo están presentando actualmente síntomas de problemas de salud mental, sino que es probable que a lo largo del tiempo aumente la incidencia de sintomatología en este grupo (Maunder et al., 2006) y a que los profesionales de la salud solicitan menos ayuda psicológica o asesoramiento debido a los prejuicios y discriminación (Zheng, 2020). 


\section{Referencias}

Acinas, P. (2012). Burn-out y desgaste por empatía en profesionales de cuidados paliativos burn-out and compassion fatigue in palliative care professionals. Sociedad Española de Medicina Psicosomática y Psicoterapia, 2(4), 2253-749. https://doi.org/10.1017/CBO9781107415324.004

Báguena, M.J., Villarroya, E., Beleña, A., Díaz, A., Roldán, C., y Reig, R. (2001) Propiedades Psicométricas de la Versión Española de la escala Revisada de Impacto del Estresor. Análisis y Modificación de Conducta, 27, 581-604.

Bastien, C., Vallieres, A., y Morín, C. (2001). Validation of the Insomnia Severity Index as an outcome measure for insomnia research. Sleep Medicine, 2(4), 297307. https://doi.org/10.1016/s1389-9457(00)00065-4

Beck, CH. T. (2011). Secondary Traumatic Stress in Nurses: A Systematic Review. Archives of Psychiatric Nursing, 25(1), 1-10.

Betancourt, M., Rodríguez, C.L., Vallecillo, I., y Sánchez, L.E. (2008). Síntomas de ansiedad y depresión en residentes de especialización médica con riesgo alto de estrés. Psiquiatría Biológica, 15, 147-152. https://doi.org/10.1016/S1134$\underline{\text { 5934(08)76485-6 }}$

Dai Y, Hu G, Xiong H, Qiu H, y Yuan X. (2020). Psychological impact of the coronavirus disease 2019 (COVID-19) outbreak on healthcare workers in China. MedRxiv (preprint). https://doi.org/10.1101/2020.03.03.20030874

Diez-Quevedo, C., Rangil, T., Sanchez-Planell, L., Kroenke, K., y Spitzer, R. L. (2001). Validation and Utility of the Patient Health Questionnaire in Diagnosing Mental Disorders in 1003 General Hospital Spanish Inpatients. Psychosomatic Medicine, 63(4), 679-686. https://doi.org/10.1097/00006842-200107000-00021

El Trece (2020). Médicos exigen ley de jubilación y equipos de bioseguridad. http://www.trece.com.py/actualidad/medicos-exigen-ley-de-jubilacion

Erdur, B., Ergin, A., Turkcuer, I., Parlak, I., Ergin, N., y Boz, B. (2006). A study of depression and anxiety among doctors working in emergency units in Denizli, Turkey. Emergency Medicine Journal, 23, 759-763. https://doi.org/10.1136/emj.2006.035071

Garcia-Campayo, J., Zamorano, E., Ruiz, M. A., Pardo, A., Perez-Paramo, M., LopezGomez, V., Freire, O., y Rejas, J. (2010). Cultural adaptation into Spanish of the generalized anxiety disorder-7 (GAD-7) scale as a screening tool. Health and Quality of Life Outcomes, 8(1), 8. https://doi.org/10.1186/1477-7525-8-8

González, C., Sánchez, Y., y Peña, G. (2018). Fatiga por compasión en los profesionales del servicio de emergencia. Dom. Cien., 4(1), 483-498. https://doi.org/10.23857/dc.v4i1.761

Harapan, H., Itoh, N., Yufika, A., Winardi, W., Keam, S., Te, H., ... Mudatsir, M. (2020). Coronavirus disease 2019 (COVID-19): A literature review. Journal of Infection and Public Health, 13(5), 667673. https://doi.org/10.1016/j.jiph.2020.03.019

IBM Corp. Released 2017. IBM SPSS Statistics for Windows, Version 25.0. Armonk, NY: IBM Corp.

Khalid, I., Khalid, T. J., Qabajah, M. R., Barnard, A. G., y Qushmaq, I. A. (2016). Healthcare Workers Emotions, Perceived Stressors and Coping Strategies During a MERS-CoV Outbreak. Clinical Medicine \& Research, 14(1), 714. https://doi.org/10.3121/cmr.2016.1303 
Kroenke, K., Spitzer, R. L., y Williams, J. B. W. (2001). The PHQ-9. Journal of General Internal Medicine, 16(9), 606-613. https://doi.org/10.1046/j.15251497.2001.016009606.x

La Nación (11 de marzo de 2020). COVID-19: Paraguay tiene 100 camas por cada millón de habitantes. https://www.lanacion.com.py/pais_edicion_impresa/2020/03/11/COVID-19paraguay-tiene-100-camas-por-cada-millon-de-habitantes/

Lai, J., Ma, S., Wang, Y., Cai, Z., Hu, J., Wei, N., ... Hu, S. (2020). Factors Associated with Mental Health Outcomes among Health Care Workers Exposed to Coronavirus Disease 2019. JAMA Network Open, 3(3), e203976. https://doi.org/10.1001/jamanetworkopen.2020.3976

Maunder, R., Lancee, W., Balderson, K., Bennett, J., Borgundvaag, B., Evans, S., ... Wasylenki, D. (2006). Long-term Psychological and Occupational Effects of Providing Hospital Healthcare during SARS Outbreak. Emerging Infectious Diseases, 12(12), 1924-1932. https://doi.org/10.3201/eid1212.060584

Ministerio de Salud Pública y Bienestar Social MSPBS (2020a). ¿Qué está haciendo el Ministerio de Salud?. https://www.mspbs.gov.py/COVID-19-actualizacion.php

Ministerio de Salud Pública y Bienestar Social MSPBS (2020b). Reportes COVID.19. https://www.mspbs.gov.py/reporte-COVID19.html

Montero, I., Aparicio, D., Gómez-Beneyto, M., Moreno-Küstner, B.,Reneses, B., Usall, J.,Vázquez-Barquero, J. (2004). Género y salud mental en un mundo cambiante. Gaceta Sanitaria, 18(Supl. 1), 175-181.

Moreno-Jiménez, B., Morante, M. E., Garrosa, E., y Rodríguez, R. (2004). Estrés traumático secundario: el coste de cuidar el trauma. Psicología Conductual, 12(2): 215-231.

O’Mahony, S., Ziadni, M., Hoerger, M., Levine, S., Baron, A., y Gerhart, J. (2018). Compassion Fatigue Among Palliative Care Clinicians. The American Journal Of $\begin{array}{lllll}\text { Hospice } & y & \text { Palliative } & \text { Care, }\end{array}$ https://doi.org/10.1177/1049909117701695

Organización Mundial de la Salud (2020a). Preguntas y respuestas sobre la enfermedad por coronavirus

(COVID-19). https://www.who.int/es/emergencies/diseases/novel-coronavirus-2019/advicefor-public/q-a-coronaviruses

Organización Mundial de la Salud (2020b). WHO Director-General's opening remarks at the media briefing on COVID-19 - 11 March 2020. https://www.who.int/dg/speeches/detail/who-director-general-s-openingremarks-at-the-media-briefing-on-COVID-19---11-march-2020

Organización Panamericana de la Salud (2020). La OMS caracteriza a COVID-19 como una pandemia. https://www.paho.org/hq/index.php?option=com_content\&view=article\&id=157 56: who-characterizes-COVID-19-as-a-pandemic\&Itemid=1926\&lang=es

Rojtenberg, S. (2001). Depresión: Una introducción. En S. Rojtengerg, Depresiones y antidepresivos (pp 1-14). Buenos Aires: Médica Panamericana

Sierra, J.C., Guillén-Serrano, V., y Santos-Iglesias, P. (2008). Insomnia Severity Index: some indicators about its reliability and validity on an older adults sample. Revista de Neurología, 47(11), 566-70. https://doi.org/10.33588/rn.4711.2008221

Spitzer, R. L., Kroenke, K., Williams, J. B. W., y Löwe, B. (2006). A Brief Measure for Assessing Generalized Anxiety Disorder. Archives of Internal Medicine, 166(10), 1092. https://doi.org/10.1001/archinte.166.10.1092 
Stamm, B.H. (1997-2005). Professional Quality of Life: Compassion Satisfaction and Fatigue Subscales, R-IV (ProQOL). http://www.proqol.org

Tam, C. W. C., Pang, E. P. F., Lam, L. C. W., y Chiu, H. F. K. (2004). Severe acute respiratory syndrome (SARS) in Hong Kong in 2003: stress and psychological impact among frontline healthcare workers. Psychological Medicine, 34(7), 1197-1204. https://doi.org/10.1017/s0033291704002247

Urzúa, A., Vera-Villarroel, P., Caqueo-Urízar, A., y Polanco, R. (2020). La Psicología en la prevención y manejo del COVID-19. Aportes desde la evidencia inicial. Terapia Psicológica, 38(1), 103-118.

Urzúa, A., Vera-Villarroel, P., Samaniego, A., Caqueo-Urízar, A., Zapata, A., y Irarrázaval, M. (2020). COVID-19 y salud mental en trabajadores de la salud en Chile. Un estudio exploratorio. Manuscrito presentado para su evaluación.

Vera-Villarroel, P. (2020). Psicología y COVID-19: un análisis desde los procesos psicológicos básicos. Cuadernos de Neuropsicología / Panamerican Journal of Neuropsychology, 14, 10-18

Weiss D. (1984). The impact of event scale-revised. En: Wilson J \& Keane T. Editores. Assessing psychological trauma and PTSD. New York, NY: Guilford Press; 2004. p. $168-89$.

Yerkes, R. M., y Dodson, J. D. (1908). The Relation of Strength of Stimulus to Rapidity of Habit-Formation. Journal of Comparative Neurology and Psychology, 18, 459482. https://doi.org/10.1002/cne.920180503

Zheng, W. (2020). Mental health and a novel coronavirus (2019-nCoV) in China. Journal of Affective Disorders, 269, 201-202. https://doi.org/10.1016/j.jad.2020.03.041

Received: $2020 / 04 / 20$ Accepted: 2020/05/05 